\title{
Unusual Tumors Obstructing the External Auditory Canal: Report of Two Cases
}

\author{
Chang-Hee Kim¹, Hye Seung Lee ${ }^{2}$, Sung-Yong Kim³ , and Jung Eun Shin ${ }^{1}$ \\ ${ }^{1}$ Departments of Otorhinolaryngology-Head and Neck Surgery, ${ }^{2}$ Pathology, ${ }^{3}$ Hemato-Oncology, Konkuk University Medical Center, \\ Konkuk University School of Medicine, Seoul, Korea
}

Received January 22, 2018

Revised March 21, 2018

Accepted May 2, 2018

Address for correspondence

Jung Eun Shin, MD, PhD

Department of Otorhinolaryngology-

Head and Neck Surgery,

Konkuk University Medical Center,

Konkuk University

School of Medicine,

120-1 Neungdong-ro, Gwangjin-gu,

Seoul 05030, Korea

Tel $+82-2-2030-7665$

Fax $+82-2-2030-5299$

E-mail 20050055@kuh.ac.kr
Primary tumors arising from the external auditory canal (EAC) are rare. We describe two cases of mass lesions within the EAC causing slowly progressive hearing loss without otorrhea or otalgia. Otoendoscopic examination demonstrated total obstruction of the EAC, and pure tone audiometry revealed conductive hearing loss. Based on the findings of the histopathologic examination, one patient was diagnosed with venous hemangioma that was treated using surgical resection, and the other patient was diagnosed with diffuse large B-cell lymphoma (DLBCL) that was treated using external-beam radiation therapy. Although primary tumors in the EAC are rare, both benign tumors such as venous hemangiomas and malignant lesions such as DLBCL should be considered as possible differential diagnoses of mass lesions in the EAC.

J Audiol Otol 2019;23(1):59-62

KEY WORDS: Venous hemangioma · Diffuse large B-cell lymphoma · External auditory canal . Conductive hearing loss.

\section{Introduction}

Mass lesions within the external auditory canal (EAC) may cause progressive conductive hearing loss, otalgia, or otorrhea. Although primary tumors involving the EAC are extremely rare, early and accurate diagnosis through histopathologic examination is important for their proper treatment.

Hemangioma is a benign vascular tumor which most commonly develops in the head and neck area. Hemangiomas can be categorized histologically into venous, cavernous, or capillary type. Capillary hemangioma is made up of tightly organized blood vessels, and the most commonly involved regions are the skin, subcutaneous tissue and mucosal surfaces of the lips. Both venous and cavernous hemangiomas are composed of larger and dilated vessels. While cavernous hemangiomas have thin-walled vascular channels which are lined by flat endothelial cells, venous hemangiomas include blood vessels with thick fibrous walls intermingled with smooth

This is an Open Access article distributed under the terms of the Creative Commons Attribution Non-Commercial License (http://creativecommons.org/licenses/by-nc/4.0/) which permits unrestricted non-commercial use, distribution, and reproduction in any medium, provided the original work is properly cited. muscle [1]. Hemangiomas arising from the EAC are rare, and the pathology is cavernous or capillary hemangioma in most cases [2].

The head and neck is the second most common area where lymphomas develop [3]. Diffuse large B-cell lymphoma (DL$\mathrm{BCL}$ ) is an aggressive malignant B-cell lymphoid neoplasm, and it accounts for approximately $30 \%$ of all malignant lymphomas. DLBCL may involve both nodal and extranodal tissues, and DLBCL accounts for approximately $50 \%$ of all extranodal head and neck lymphomas [4]. DLBCLs originating from the EAC are extremely rare, and their clinical and pathological characteristics have not been extensively discussed.

In this report, we describe two cases of EAC-obstructing masses diagnosed as venous hemangioma in one patient and DLBCL in the other. A literature review regarding venous hemangiomas and DLBCLs originating from the EAC follows the case report.

\section{CASE REPORT}

\section{Case 1}

A 55-year-old woman visited our clinic with a symptom of 
slowly progressing hearing loss on the right side. The patient reported bleeding from the right ear after ear picking 2 weeks ago, and did not complain of otalgia, otorrhea, or dizziness. She had no previous history of otorrhea, otologic surgery, or recent ear or brain trauma. Otoendoscopic examination revealed a reddish mass obstructing the EAC (Fig. 1A). The tympanic membrane was not visible because of the EAC mass lesion. Pure tone audiometry revealed conductive hearing loss with a $35-\mathrm{dB}$ air-bone gap on the right side. High-resolution temporal bone computed tomography revealed a soft-tissue mass with mild enhancement without bony erosion or destruction in the right EAC (Fig. 1C, D). No evidence of extension into the middle ear cavity was noted. We performed an excisional biopsy of the mass via the transcanal approach under a surgical microscope. The mass originated from the superior wall of the EAC, and was confined within the EAC. The mass was completely removed, and a skin graft was not required. Histopathological examination revealed a proliferation of thick-walled vessels that were variably dilated in the myxoid stroma (Fig. 1B). After 16 months postoperatively, the patient has shown no evidence of recurrence (Fig. 3A).

\section{Case 2}

A 76-year-old woman was referred to our clinic with the left EAC mass lesion. The patient had been experiencing ear fullness, hearing loss, otorrhea, and otalgia on the left side for 2 months. She had visited another hospital before being referred to our clinic, and had undergone incisional biopsy that revealed a histologic diagnosis of a ruptured epidermal cyst. She did not report headache or dizziness, and had no previous history of ear disease, ear or brain trauma, otologic surgery, or lymphoproliferative disorder. Otoendoscopic examination revealed a reddish polypoid mass obstructing the left EAC (Fig. 2A), which made the tympanic membrane invisible. Conductive hearing loss with a $35-\mathrm{dB}$ air-bone gap was noted on pure tone audiometry. High-resolution temporal bone computed tomography demonstrated an infiltrative, enhancing soft-tissue mass with complete obstruction of the left $\mathrm{EAC}$ and bony erosion of the inferior wall of the EAC; moreover, the mass lesion extended to the left parotid space (Fig. $2 \mathrm{C}, \mathrm{D})$. Under the clinical impression of a malignant soft-tissue tumor, we performed an incisional biopsy. Histopathological examination revealed diffuse proliferation of abnormally large lymphoid cells (Fig. 2B). Immunohistochemical staining tests showed that the atypical lymphoma cells were posi-
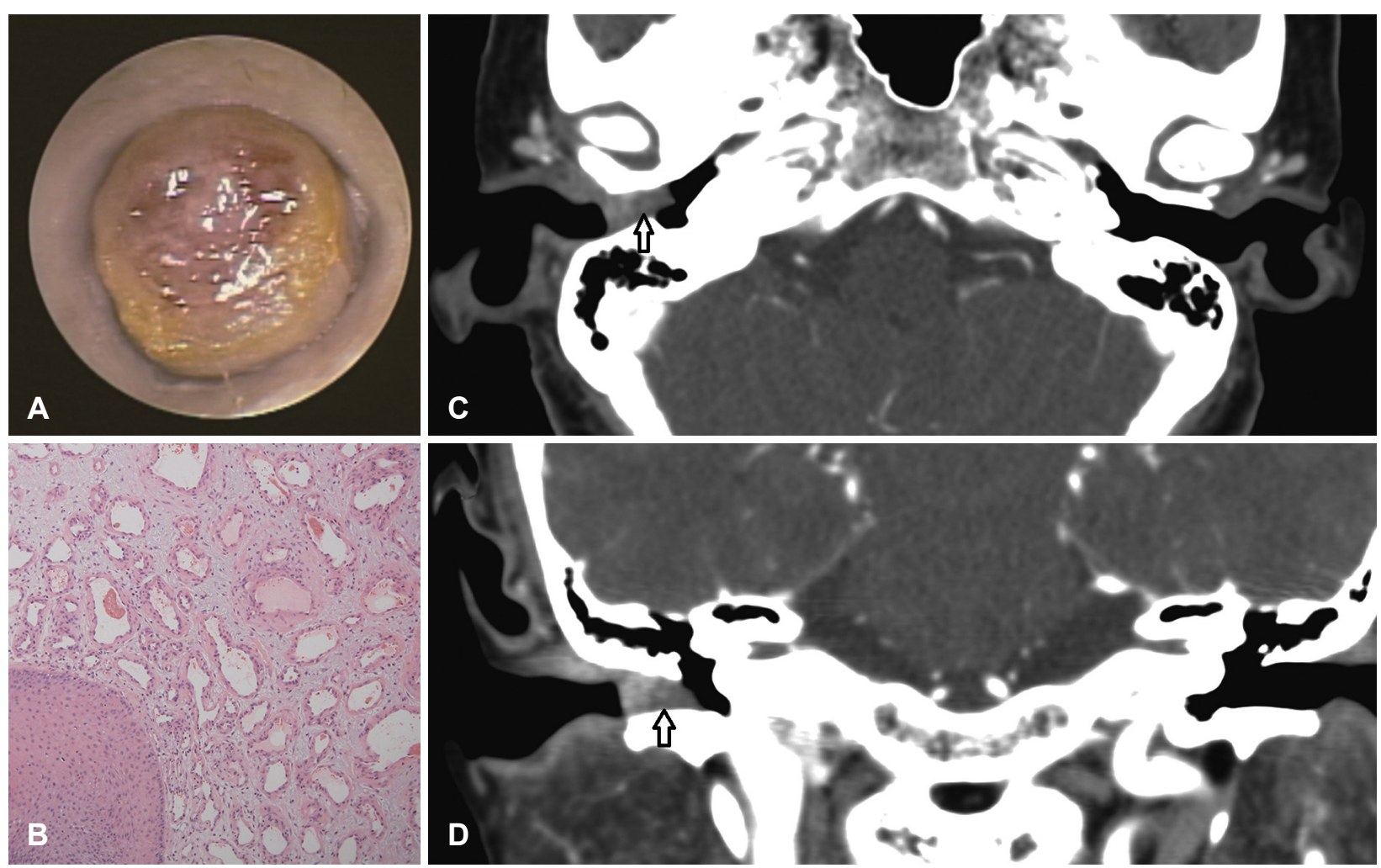

Fig. 1. Otoendoscopic examination reveals a reddish mass lesion blocking the external auditory canal (EAC) (A). Histologic examination demonstrates large and irregular blood vessels with thick vascular walls embedded in a myxoid stroma (hematoxylin \& eosin, $X$ 100) (B). High-resolution temporal bone computed tomography reveals a soft-tissue mass with enhancement in the right EAC without bony erosion in the axial (C) and coronal (D) sections (arrows). 

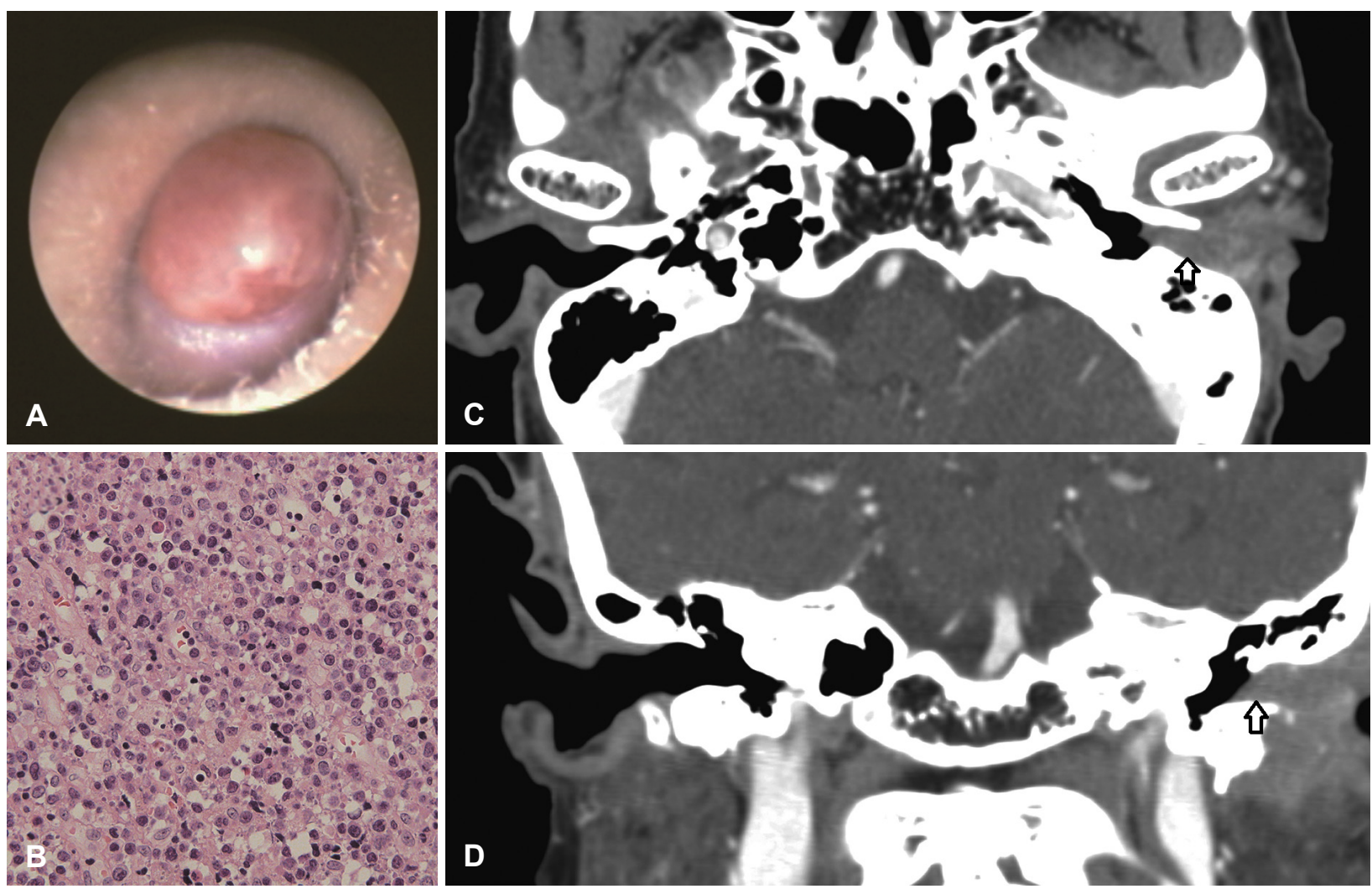

Fig. 2. Otoendoscopic examination reveals a red-colored mass obstructing the left external auditory canal (EAC) (A). Histologic findings demonstrate diffuse infiltration of large-sized lymphoid cells (hematoxylin \& eosin, $\times 400)(B)$. High-resolution temporal bone computed tomography reveals an enhancing soft-tissue mass in the left EAC infiltrating into the left parotid space (arrows) (C, axial view; D, coronal view).

Fig. 3. Post-treatment otoendoscopic findings in case $1(\mathrm{~A})$ and $2(\mathrm{~B})$.
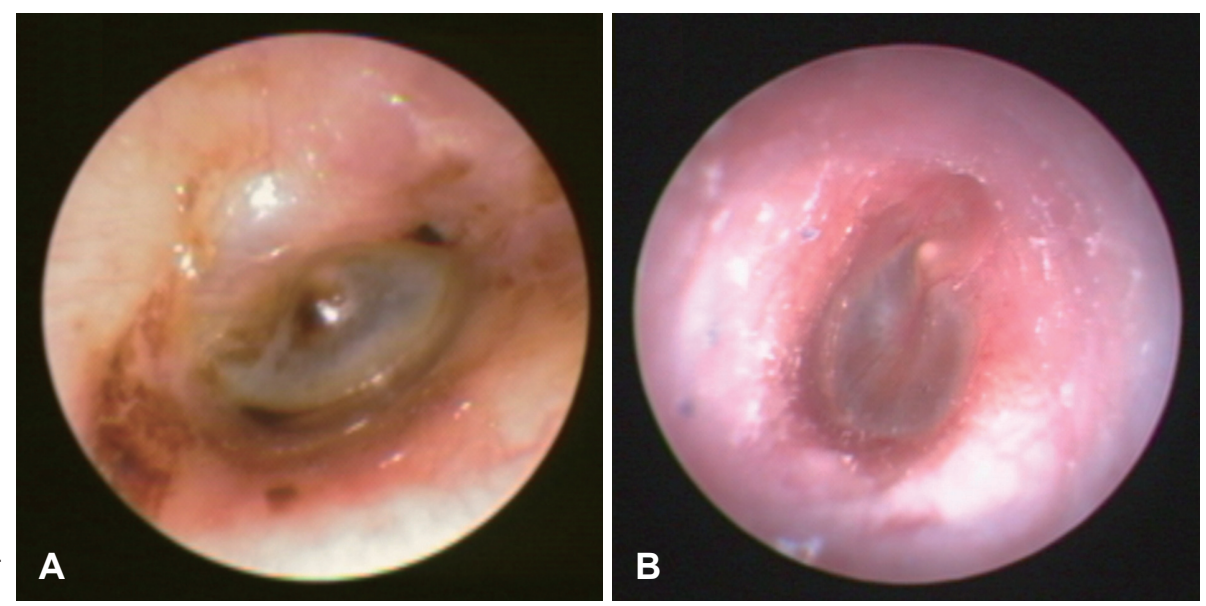

tive for CD20, CD79a, BCL2, BCL6, and Mum-1, and negative for CD3 and CD10, thereby suggesting a diagnosis of DLBCL. Therefore, clinical staging was performed using brain magnetic resonance imaging, computed tomography of the abdomen and chest, whole-body positron emission tomography, and bone marrow biopsy, which revealed no other localization of the lymphoma. The patient was treated with local radiotherapy of $50.4 \mathrm{~Gy}$, which reduced the size of the tumor dramatically (Fig. 3B). No measurable remaining mass lesion was observed on follow-up CT 6 months after treatment.

\section{Discussion}

Hemangioma is one of the most common soft tissue tumors, and can be classified into capillary, cavernous, and venous. Arteriovenous malformation, which is characterized by 
an interconnected complex of arterial and venous structures, can also be included as a category of hemangioma $[5,6]$. Twenty-six cases with hemangioma arising from the EAC have been reported in the literature, and, in most of these cases, the histologic type was capillary or cavernous [2]. To the best of our knowledge, ours is the first reported case of a venous hemangioma in the EAC. Patients with EAC hemangiomas may present with symptoms such as hearing loss, aural fullness, tinnitus, or bloody otorrhea. However, most patients do not show any symptoms, and are diagnosed incidentally because the size of the tumors is small, ranging from $5 \mathrm{~mm}$ to $3 \mathrm{~cm}$ in diameter [7]. The differential diagnosis for EAC hemangiomas may include cholesteatoma with an aural polyp, arteriovenous malformation, granulation tissue, glomus tumor, and carcinoma. Histologic examination is mandatory for the correct diagnosis, and high-resolution temporal bone computed tomography is of great help in delineating the tumor involvement. In some cases, angiography is necessary [8]. Complete surgical excision is the treatment of choice.

Lymphomas arising from the EAC are extremely rare. To our knowledge, only 10 cases of primary lymphomas arising from the EAC have been reported [9]. Besides the EAC, primary lymphomas have been reported to develop in the internal auditory canal [10], middle ear [11], and mastoid cavity [12] in the temporal bone area, and among these, the mastoid and middle ear cavity are known as the most common primary focus of origin [13]. Since primary lymphoma in the EAC has highly nonspecific clinical presentations, this disease can often be misdiagnosed as otitis externa [14]. Otalgia, aural fullness, and hearing loss are reported to be the most common presenting symptoms [15]. Taken together with its rarity, the absence of disease-specific symptoms or signs makes early diagnosis difficult. Moreover, an incisional biopsy may lead to a misdiagnosis or delayed diagnosis as in one of our patients; therefore, repeated biopsy is sometimes recommended in cases with a high suspicion of malignancy [14]. After diagnosis, our patient received external-beam radiation therapy only, and achieved a favorable response.

Inflammatory lesions such as cholesteatoma, aural polyp and granulation tissue are more common diagnoses than the tumorous lesions when mass-like lesions are observed in the EAC. However, malignant tumors such as squamous cell carcinoma, lymphoma, basal cell carcinoma, adenoid cystic carcinoma, adenocarcinoma, ceruminous carcinoma, and malignant fibrous histiocytoma, or benign tumors such as hemangioma, pleomorphic adenoma, and cylindroma should be considered as a differential diagnosis.

In conclusion, although primary tumors arising from the EAC are extremely rare, both benign tumors such as venous hemangiomas and malignant tumors such as DLBCL should be considered as differential diagnoses to ensure proper management of mass lesions in the EAC.

\section{Acknowledgments}

This paper was written as part of Konkuk University's research support program for its faculty on sabbatical leave in 2014.

\section{Conflicts of interest}

The authors have no financial conflicts of interest.

\section{REFERENCES}

1) Beham A, Fletcher CD. Intramuscular angioma: a clinicopathological analysis of 74 cases. Histopathology 1991;18:53-9.

2) Rutherford KD, Leonard G. Hemangiomas of the external auditory canal. Am J Otolaryngol 2010;31:384-6.

3) Merkus P, Copper MP, Van Oers MH, Schouwenburg PF. Lympho$\mathrm{ma}$ in the ear. ORL J Otorhinolaryngol Relat Spec 2000;62:274-7.

4) Sabattini E, Bacci F, Sagramoso C, Pileri SA. WHO classification of tumours of haematopoietic and lymphoid tissues in 2008: an overview. Pathologica 2010;102:83-7.

5) Kim SB, Lee HH. Capillary hemangioma of the tympanic membrane and external auditory canal. J Craniofac Surg 2017;28:e231-2.

6) Jang CH, Choi HS, Hong YS, Cho YB. Cavernous hemangioma of the tympanic membrane. Clin Exp Otorhinolaryngol 2011;4:109-11.

7) Yang TH, Chiang YC, Chao PZ, Lee FP. Cavernous hemangioma of the bony external auditory canal. Otolaryngol Head Neck Surg 2006; 134:890-1.

8) Limb CJ, Mabrie DC, Carey JP, Minor LB. Hemangioma of the external auditory canal. Otolaryngol Head Neck Surg 2002;126:74-5.

9) Bruschini L, De Vito A, Fortunato S, Pelosini M, Cervetti G, Petrini $\mathrm{M}$, et al. A case of primary non-Hodgkin's lymphoma of the external auditory canal. Case Rep Otolaryngol 2013;2013:138397.

10) Ryou N, Ko DY, Jun HJ, Chae SW. Lymphoma of the internal auditory canal presenting as facial palsy, vertigo, and hearing loss. J Int Adv Otol 2015;11:262-3.

11) Li B, Liu S, Yang H, Wang W. Primary T-cell lymphoblastic lymphoma in the middle ear. Int J Pediatr Otorhinolaryngol 2016;82:1922.

12) Tucci DL, Lambert PR, Innes DJ Jr. Primary lymphoma of the temporal bone. Arch Otolaryngol Head Neck Surg 1992;118:83-5.

13) Hersh SP, Harrison WG, Hersh DJ. Primary B cell lymphoma of the external auditory canal. Ear Nose Throat J 2006;85:597-9.

14) Fish BM, Huda R, Dundas SA, Lesser TH. B-cell lymphoma of the external auditory meatus. J Laryngol Otol 2002;116:39-41.

15) Ogawa S, Tawara I, Ueno S, Kimura M, Miyazaki K, Nishikawa H, et al. De novo CD5-positive diffuse large B-cell lymphoma of the temporal bone presenting with an external auditory canal tumor. Intern Med 2006;45:733-7. 